\title{
Patient with a short history of hemoptysis, fever and melena
}

\section{Mohammad Mizanur Rahman, Mohammed Mosleh Uddin, Mostafil Karim, Monwar Tarek and Farzana Shabnam}
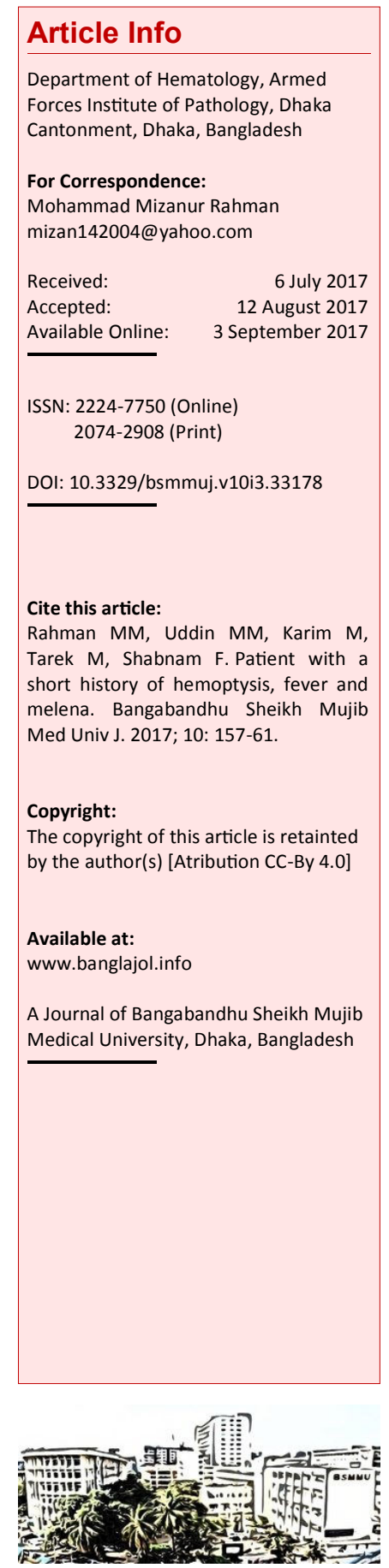

\section{Presentation of Case}

Dr Farzana Shabnam: A 27 year old man was evaluated in the emergency and casualty room of a military hospital because of high fever, hemoptysis, melena, generalized weakness and burning sensation during micturition.

The patient had been well until one month before presentation to this hospital, when he developed coughing up blood which was mixed with sputum. Blood was fresh, bright red in color and small in amount. Two weeks later he developed fever which was high grade in nature, continued, not associated with chills and rigor but subsided after taking tablet paracetamol. At the same time he noticed of passing black tarry stool. There was no history of weight loss and jaundice. He had no past history of hospital admission for any illness. He did not give any recent travel history to Hill Tracts (South-East part of Bangladesh) where malaria infection is endemic. The patient is an engine mechanic serving in the Bangladesh Navy.

On examination in the emergency and casualty room, the temperature was $102^{\circ} \mathrm{F}$, the blood pressure $130 / 80 \mathrm{mmHg}$, the pulse 85 beats per min and the respiratory rate 18 breaths per min. However, the patient appeared ill-looking and anxious because of hemoptysis and melena. He also had moderate anemia. The examination of the cardiovascular, respiratory, genito-urinary, nervous and musculoskeletal systems was normal. But the examination of the gastrointestinal system revealed tenderness in the right hypochondrium. Examination of the hemopoietic system also revealed moderate anemia, bony tenderness and mild hepatomegaly but no lymphadenopathy, subconjunctival hemorrhage or purpuric rash was found.

The attending physician also sent blood and urine specimens for routine investigations and requested for $\mathrm{X}$-ray chest $(\mathrm{P} / \mathrm{A}$ view) and ultrasonography of the whole abdomen.

After evaluating the clinical data, physical findings and the investigation results especially the complete blood count report, the attending physician of the emergency and casualty room admitted the patient into Hematology Ward. The medical officer in charge of the Hematology ward after analyzing all the clinical and physical findings as well as the investigation results so far received made the provisional diagnosis of acute leukemia and advised for bone marrow morphological examination, immunophenotyping and molecular study for acute myeloid leukemia.

\section{Provisional Diagnosis}

Acute leukemia

\section{Differential Diagnosis}

Dr Mohammad Mizanur Rahman: In the development of the differential diagnosis, several features of the patient's presentation informed. First, the illness developed suddenly within a very short course of time which indicates an acute nature of the disease. Second, he presented with generalized weak-ness with moderate pallor, high fever subsided after taking non-steroidal anti-inflammatory drugs as well as hemorrhagic manifestation and bony tenderness indicating the involvement of three cell lineages of hemopoietic system. Finally, most of the presenting features are important clues to the diagnosis of acute leukemia; these include moderate anemia, high temperature, hemoptysis and melena. After evaluating the patient's presenting features, I had considered the following differential diagnosis.

\section{Infectious Mononucleosis}

Infectious mononucleosis is a clinical disorder distinguished by pharyngitis, cervical lymph node enlargement, fever and fatigue which results most often from primary Epstein-Barr virus infection. 1 Epstein-Barr virus, a lymphocryto-virus and a member of the $\gamma$-herpes virus family, infects at least $90 \%$ of the population worldwide, the majority of whom have no recognizable illness. 2 As the patient presented with generalized weakness, moderate anemia, fever, mild hepatomegaly and bleeding manifestation, therefore, these features are sugges- 


\begin{tabular}{|c|c|c|}
\hline \multicolumn{3}{|c|}{ Table I } \\
\hline \multicolumn{3}{|c|}{ Laboratory data } \\
\hline Variable & Reference range, adult & In the Medical Ward \\
\hline Hemoglobin $(\mathrm{g} / \mathrm{dL})$ & $13.0-17.0$ & 8.7 \\
\hline Hematocrit (L/L) & $0.45-0.50$ & 0.26 \\
\hline $\mathrm{RBC}\left(\times 10^{12} / \mathrm{L}\right)$ & $4.5-5.5$ & 3.4 \\
\hline Mean cell volume (fL) & $86-101$ & 89 \\
\hline White cell count $\left(\times 10^{9} / \mathrm{L}\right)$ & $4.0-10.0$ & 15.8 \\
\hline \multicolumn{3}{|l|}{ Differential count $(\%)$} \\
\hline Neutrophils & $40-80$ & 07 \\
\hline Lymphocytes & $20-40$ & 10 \\
\hline Monocytes & $2-10$ & 02 \\
\hline Eosinophils & $1-6$ & 01 \\
\hline Basophils & $<1-2$ & 00 \\
\hline Blasts & 00 & 80 \\
\hline Platelet count $\left(\times 10^{9} / \mathrm{L}\right)$ & $150-410$ & 15 \\
\hline E.S.R (mm in 1st hour) & $0-22$ & 110 \\
\hline
\end{tabular}

tive of infectious mononucleosis. But the absence of cervical lympadenopathy and/or splenomegaly is against the diagnosis of infectious mononucleosis.

\section{Aplastic Anemia}

Aplastic anemia is a life-threatening bone marrow failure disorder which, if untreated, is associated with very high mortality. 3 This patient presented with the generalized weakness due to moderate anemia, high fever and bleeding manifestation. So, these findings are in favor of the aplastic anemia. However, presence of bony tenderness and absence of superficial bleeding are the features contradicting the diagnosis of aplastic anemia.

\section{Myelodysplastic Syndrome}

The myelodysplastic syndrome is a heterogeneous group of malignant hematopoietic disorder characterized by dysplastic changes in one or more cell lineages, ineffective hematopoiesis and a variable predilection to development of acute myeloid leukemia. 4 Patient with myelodysplastic syndrome may present with the clinical features of anemia, thrombocytopenia and/or neutropenia. $\frac{5}{\text { In the }}$ present case, patient presented with the clinical features of anemia, e.g., generalized weakness, neutropenia such as high fever and bleeding manifestation such as hemoptysis and melena which are in favor of myelodysplastic syndrome but age and presence of bony tenderness are against the diagnosis.

Dr Mohammed Mosleh Uddin: After evaluating the patient's presenting features, physical findings and the laboratory test results which is shown in Table I,



Figure 1: Photograph of peripheral blood film (A) and bone marrow (B)

I did the bone marrow aspiration and repeat the complete blood count and peripheral blood film examination. I also sent bone marrow samples overseas for immunophenotyping and cytogenetic/ molecular study for acute myeloid leukemia. Peripheral blood film showed rouleaux formation of red cells, significant number of promyelocytes (approximately $80 \%$ of the white cell differentials) and platelets were reduced, suggesting the diagnosis of acute myeloid leukemia (Figure 1A).

Bone marrow aspirates also showed grossly hypercellular marrow with increased myeloid Erythroid ratio. Erythropoiesis was depressed and normoblastic. Granulopoiesis was hyperactive and showed numerous promyelocytes (approximately $80 \%$ of the white cell differentials). Megakaryocytes were scanty. Lymphocytes, histiocytes and plasma cells are also seen. The bone marrow finding confirmed the morphological diagnosis of acute promyelocytic leukemia (Figure 1B)

Bone marrow sample which was sent overseas for quantitative assay of PML-RARA and received result was reported as PML/RARA: $t(15 ; 17)$ (q24; q21): $35.4 \%$.

\section{Dr. Shabnam's Diagnosis}

Acute myeloid leukemia with t $(15 ; 17)$; PML-RARA.

\section{Discussion}

Dr. Rahman: Acute myeloid leukemia with $\mathrm{t}(15 ; 17)$; PML-RARA is the M3 subtype of acute myeloid leukemia.6 Acute promyelocytic leukemia was first 
characterized in 1957 by French and Norwegian physicians as hyperacute fatal illness with a median survival time of less than a week. $\frac{7,8}{2}$ The disease is caused by balanced reciprocal translocation between long arms of chromosome 15 and 17 and this molecular basis of the disease leads to targeted therapy with all-trans retinoic acid (ATRA) in 1988 with excellent cure rate. 9 Today, prognoses have drastically improved; 10-year survival rates are estimated to be approximately $77 \% . \underline{10}$

In $95 \%$ of cases of acute promyelocytic leukemia, retinoic acid receptor-alpha (RARA) gene on chromosome 17 is involved in a reciprocal translocation with the promyelocytic leukemia gene (PML) on chromosome 15, a translocation denoted as $\mathrm{t}(15 ; 17)$ (q22;q12).10 The RARA is dependent on retinoic acid for regulation of transcription. $?$

Eight other rare gene rearrangements have been described in acute promyelocytic leukemia fusing RARA to promyelocytic leukemia zinc finger (PLZF), nucleophosmin (NPM1), nuclear matrix associated (NUMA1), signal transducer and activator of transcription 5b (STAT5B), protein kinase A regulatory subunit 1a (PRKAR1A), factor interactting with PAPOLA and CPSF1 (FIP1L1), BCL6 corepressor (BCOR) or oligonucleotide/oligosaccharide-binding fold containing $2 \mathrm{~A}$ (OBFC2A) genes. Some of these rearrangements are ATRA-sensitive or have unknown sensitivity to ATRA because they are so rare; STAT5B/RARA and PLZF/RARA are known to be resistant to ATRA.?

The fusion of PML and RARA results in expression of a hybrid protein with altered functions. This fusion protein binds with enhanced affinity to sites on the cell's DNA, blocking transcription and differentiation of granulocytes. It does so by enhancing interaction of nuclear co-repressor (NCOR) molecule and histone deacetylase (HDAC). Although the chromosomal translocation involving RARA is believed to be the initiating event, additional mutations are required for the development of leukemia. $?$

RARA/PLZF gene fusion produces a subtype of acute promyelocytic leukemia that is unresponsive to tretinoin therapy and less responsive to standard anthracycline chemotherapy hence leading to poorer long-term outcomes in this subset of patients. $\underline{11}$

Considering the pathogenesis of acute promyelocytic leukemia and laboratory data of this patient, we consider ATRA and arsenic trioxide is the standard protocol. Therefore, this patient was managed with ATRA and arsenic trioxide with necessary supportive measures. After the completion of induction therapy, peripheral blood film and bone marrow examination were done. Morphological as well as molecular (PML-RARA) testing were re-assessed and found that the patient had under- gone complete hematological remission. As the patient underwent complete hematological remission, he was discharged and advised to come for admission for ten days in every month for maintenance therapy (injection arsenic trioxide, $8 \mathrm{mg}$ [0.15 mg/ kg body weight/day] in $100 \mathrm{~mL}$ normal saline for 10 days in every month) for six months.

Dr. Md. Monirul Islam: Why arsenic trioxide is used for the treatment of this patient?

Dr. Rahman: Arsenic trioxide is used as a differentiating agent. $\underline{11}$

Dr. Mostafil Karim: We know arsenic is a carcinogen. Will its use augment the disease?

Dr. Shabnam: Carcinogenicity of any carcinogen depends on its dose and duration of administration. Chronic arsenic poisoning is known as arsenicosis. $\underline{12}$ Long-term ingestion of arsenic trioxide either in drinking water or as a medical treatment can lead to skin cancer. 13 Arsenic trioxide induces cancer cells to undergo apoptosis. $\underline{14}$ Arsenic trioxide is a chemotherapeutic agent for the treatment of acute promyelocytic leukemia that is unresponsive to first line agents, namely ATRA or in combination of both ATRA and arsenic trioxide.15, 16 As arsenic trioxide will be given for six months only, hopefully it will not enhance the disease process rather it will help to undergo remission.

Dr. Md. Shahidul Islam: What is differentiation syndrome?

Dr. Rahman: ATRA syndrome and differentiation syndrome are synonyms. This syndrome develops during the use of ATRA. This is associated with the development of dyspnea, fever, weight gain, peripheral edema and is treated with dexamethasone. The etiology of retinoic acid syndrome has been attributed to capillary leak syndrome caused by cytokines released from the differentiating promyelocytes.11

Dr. Mahjabin: What are other varieties of acute myeloid leukemia with $\mathrm{t}(15 ; 17)$; PML-RARA?

Dr. Mohammed Mosleh Uddin: Eight other rare gene rearrangements have been described in acute promyelocytic leukemia fusing $R A R A$ to promyelocytic leukemia zinc finger (PLZF also known as ZBTB16), nucleophosmin (NPM1), nuclear matrix associated (NUMA1), signal transducer and activetor of transcription $5 \mathrm{~b}(S T A T 5 B)$, protein kinase $\mathrm{A}$ regulatory subunit 1a (PRKAR1A), factor interactting with PAPOLA and CPSF1 (FIP1L1), BCL6 co repressor $(B C O R)$ or oligo-nucleotide/oligosaccharide-binding fold containing 2A (OBFC2A also known as NABP1) genes. $-\frac{7}{2}$

Dr. Azad: Is there any predilection of acute promyelocytic leukemia to any particular age group and geographical area? 
Dr. Rahman: Among Latinos, the incidence of acute promyelocytic leukemia is $20-28 \%$ of acute myeloid leukaemia. 6 Acute promyelocytic leukemia most commonly occurs in young age group than other FAB subtypes of acute myeloid leukemia and it has several distinctive morphologic, biologic and cytogenetic characteristics. 17

Dr. A. K. M. Abu Yusuf: How will you follow-up this patient?

Dr. Monowar Tarek: After stable remission is induced, the standard of care is to undergo 2 years of maintenance chemotherapy with methotrexate, mercaptopurine and ATRA. During this time, the patient should be investigated with peripheral blood count every month and bone marrow examination every after 6 months. .11

Dr. A. K. M. Abu Yusuf: What are the unique features of acute promyelocytic leukemia?

Dr. Rahman: Unique features are that it occurs in relatively younger age; there is balanced reciprocal translocation between chromosome 15 and 17; more prone to develop disseminated intravascular coagulation; it is a medical emergency and needs urgent therapeutic intervention. Also its prognosis is good in comparison to other FAB subtypes.18 Clinically it is usually characterized by life threatening hemorrhagic complication that may cause disseminated intravascular coagulation (DIC) and hyperfibrinolysis. $\underline{19}$

Dr. Lutfunnahar Khan: Is there any other newer drugs used for the treatment of acute promyelocytic leukemia?

Dr. Rahman: Some evidence supports the potential therapeutic utility of histone deacetylase inhibitors such as valproic acid or vorinostat in treating acute promyelocytic leukemia. 20 According to one study, a cinnamon extract has effect on the apoptotic process in acute myeloid leukemia HL-60 cell.21 These drugs are all investigational agents.

Dr. Arif Ahmed Khan: What is the curative treatment of this patient?

Dr. Uddin: Both allogeneic (ALLO) and autologous (AUTO) hematopoietic stem cell transplantation (HSCT) are effective in acute myeloid leukemia, but their role in acute promyelocytic leukemia is not clear because treatment outcome with ATRA, arsenic trioxide and chemotherapy is excellent and results in apparent cure in about $70-80 \%$ of patients. $\underline{.2}$

Dr. Yusuf: How long maintenance therapy will be continued and what type of drug will be given?

Dr. Rahman: Maintenance therapy with ATRA should be continued for at least two year.22

Dr. Debashish Saha: What is the mode of death of such patient?
Dr. Rahman: About one-third of patient died of bleeding manifestation, usually intracerebral hemorrhage. This type of hemorrhage may develop either at presentation or immediately after the initiation of induction therapy. $\underline{23}$

\section{Final Diagnosis}

Acute myeloid leukemia with $\mathrm{t}(15 ; 17)$; PMLRARA.

\section{References}

1. Ebell MH. Epstein-Barr virus infectious mononucleosis. Am Fam Physician. 2004; 70: 1279-87.

2. Balfour HH, Dunmire KS, Hogquist AK. Infectious mononucleosis. Clin Transl Immunol. 2015; 4: e33.

3. Young SN, Scheinberg P, Calado TR. Aplastic anemia. Curr Opin Hematol. 2008; 15: 162-68.

4. Guidelines for the diagnosis and management of adult myelodysplastic syndrome. British Committee for Standards in Hematology, 2013.

5. Greenberg PL, Tuechler H, Schanz J, Sanz G, Garcia -Manero G. Revised international prognostic scoring system for myelodysplastic syndromes. Blood 2012; 120: 2454-65.

6. Hassan R, Abdullah AD, Husin A, Abdullah WZ, Mustaffa R, Ghazali S, Chen Yong ANG, Baba AA. Characterizing PML/RARAa isoforms of acute promyelocytic leukemia (APL) in Malay patients. Bangladesh J Med Sci. 2014; 13: 311-15.

7. Sainty D, Liso V, Cantu-Rajnoldi A. Molecular cytogenetic diagnosis in haematological malignancies: A new morphologic classification system for acute promyelocytic leukemia distinguishes cases with underlying PLZF/RARA gene rearrangements. Blood 2000; 96: 1287-96.

8. Tallman MS, Altman JK. Curative strategies in acute promyelocytic leukemia. Hematology Am Soc Hematol Educ Program. 2008: 391-99.

9. Hillestad, LK. Acute promyelocytic leukemia. Acta Med Scand. 1957; 159: 189-94.

10. Coombs CC, Tavakkoli M, Tallman MS. Acute promyelocytic leukemia: Where did we start, where are we now, and the future. Blood Cancer J. 2017; 5: e304.

11. Fenaux P, Chastang C, Chevret S. A randomized comparison of all transretinoic acid (ATRA) followed by chemotherapy and ATRA plus chemotherapy and the maintenance therapy in newly diagnosed acute promyelocytic leukemia. The European APL group. Blood 1999; 94: 1192-200.

12. Gore SD, Gojo I, Sekeres MA. Single cycle of arsenic trioxide-based consolidation chemotherapy spares anthracycline exposure in the primary management 
of acute promyelocytic leukemia. J Clin Oncol. 2010; 28: 1047-53.

13. Adès L, Guerci A, Raffoux E, Sanz M, Chevallier P. European APL Group, March 2010. Very long-term outcome of acute promyelocytic leukemia after treatment with all-trans retinoic acid and chemotherapy: The European APL Group experience. Blood 2009; 115: 1690-96.

14. Emsley J. Arsenic. The elements of murder: A history of poison. London, Oxford University Press, 2006, pp 93-197.

15. Soignet SL, Frankel SR, Douer D, Tallman MS, Kantarjian H, Calleja E, Stone RM, Kalaycio M, Scheinberg DA. United States multicenter study of arsenic trioxide in relapsed acute promyelocytic leukemia. J Clin Oncol. 2001; 19: 3852-60.

16. Antman KH. Introduction: The history of arsenic trioxide in cancer therapy. Oncologist 2001; 6 (Suppl 2): 1-2.

17. Ara T, Khan MA, Afrose $S$, Nazneen $H$, Ali M, Islam MM, Akther M, Nazim Uddin AYM. A case report and literature review on pregnancy with acute promyelocytic leukemia with DIC. J Dhaka Med Coll. 2014; 23: 262-65.
18. Kabir MA, Rahman MM, Mowla MGS, Moniruzzaman M, Rahim A, Jahan S, Ahsan NMH. Acute promyelocytic leukemia: An experience with 6 cases. J Med. 2011; 12: 139-41.

19. Rauta S, Sahoo AK. Acute promyelocytic leukemia in a 17-year old male presenting as intracranial hemorrhage. Bangladesh J Med Sci. 2014; 132: 8890.

20. He LZ, Tolentino T, Grayson P. Histone deacetylase inhibitors induce remission in transgenic models of therapy-resistant acute promyelocytic leukemia. J Clin Invest. 2001; 108: 1321-30.

21. Assadollahi V, Parivar K, Roudbari NH, Khalatbary AR, Motamedi M, Ezatpour B, Dashti GR. The effect of aqueous cinnamon extract on the apoptotic process in acute myeloid leukemia HL-60 cells. Adv Biomed Res. 2013; 2: 25.

22. Nabhan C, Mehta J, Tallman MS. The role of bone marrow transplantation in acute promyelocytic leukemia. Bone Marrow Transplantation 2001; 28: 219-26.

23. Lo-Coco F, Avvisati G, Vignetti M. Retinoic acid and arsenic trioxide for acute promyelocytic leukemia. N Engl J Med. 2013; 369: 111-21. 